\title{
VOLUME MOLAR EXCESSO DE SOLUÇÕES LÍQUIDAS BINÁRIAS DE GLICEROL + ÁLCOOIS A DIFERENTES TEMPERATURAS E À PRESSÃO ATMOSFÉRICA
}

\author{
V. B. VICENTE ${ }^{1}$, R. B. TÔRRES ${ }^{2}$ \\ ${ }^{1}$ FEI, Departamento de Engenharia Mecânica, \\ ${ }^{2}$ FEI, Departamento de Engenharia Química, \\ E-mail para contato: belchior@fei.edu.br
}

\begin{abstract}
RESUMO - Neste estudo, foram determinados dados experimentais de densidades de soluções líquidas binárias de glicerol + metanol, ou + etanol, ou + 1-propanol, ou + 2-propanol, ou + 1-butanol, em toda faixa de composição, a diferentes temperaturas e à pressão atmosférica. As temperaturas estudadas foram: $288,15 \mathrm{~K}$, $293,15 \mathrm{~K}, 298,15 \mathrm{~K}, 303,15 \mathrm{~K}$ e $308,15 \mathrm{~K}$. As densidades foram determinadas usando um densímetro de oscilação mecânica da marca Anton Paar (Modelo DMA 4500). Os resultados experimentais foram usados para calcular o volume molar excesso, $V_{\mathrm{m}}^{\mathrm{E}}$, dos sistemas estudados, os quais foram correlacionados através de um polinômio de Redlich-Kister. Os valores do volume molar excesso foram negativos, em toda faixa de composição, para todos os sistemas, e aumentaram com o aumento da temperatura e da cadeia carbônica, seguindo a ordem: metanol $>$ etanol $>2$ propanol >1-propanol > 1-butanol.
\end{abstract}

\section{INTRODUÇÃO}

O estudo de grandezas excesso é um tema clássico e de grande relevância na termodinâmica química de soluções, sobretudo, porque a indústria química necessita de valores dessas grandezas para projetos de processos químicos. Além disso, dados de densidades e propriedades volumétricas são de grande importância para tentar compreender as possíveis interações intermoleculares presentes nos sistemas estudados.

$\mathrm{O}$ uso de novas tecnologias em equipamentos de medidas de densidade possibilitou o avanço do estudo de grandezas volumétricas devido à grande precisão com que essas são determinadas utilizando pequenos volumes de amostras.

Dentre as grandezas excesso, o volume molar excesso é de grande importância, uma vez que o seu comportamento está associado a efeitos físicos, químicos e estruturais. Em virtude da complexidade associada ao seu comportamento, juntamente com a sua pequena magnitude e relativa facilidade de obtê-lo com rapidez e grande precisão, utilizando pequenas quantidades de 
amostra, faz do volume molar excesso uma grandeza de grande utilidade no desenvolvimento e teste de modelos e teorias de solução.

Glicerol pertence à classe de solventes que apresentam ligações de hidrogênio em redes tridimensionais, assim como a água e os dióis (Towey et al., 2011; Champeney et al., 1986; Chelli et al., 1999a; Chelli, et al., 1999b; Chelli et al., 2000; Van Koningsveld, 1968; Garawia, 1987; Dashnau, 2006; Marcus, 2000). Essas estruturas permitem aos sistemas líquidos algumas propriedades específicas, como baixa compressibilidade isotérmica e expansividade térmica isobárica, volume livre relativamente grande e alta viscosidade, entre outras. A molécula do glicerol possui três grupamentos hidroxilas que permitem formar ligações de hidrogênio inter e intramoleculares. O glicerol é capaz de formar 126 conformações como constatado em diferentes estudos de dinâmica molecular (MD) (Chelli et al., 1999a; Chelli, et al., 1999b; Callam et al., 2001).

Glicerol e alcoóis são importantes solventes na indústria farmacêutica e de alimentos. Eles formam entre si sistemas líquidos binários completamente miscíveis. Sistemas contendo glicerol e alcoóis podem ser usados como um fluido de mistura entre óleo pesados e hidrocarbonetos ou dióxido de carbono no processo de recuperação de óleo por extração a vapor. A mistura desses solventes com óleo reduz a viscosidade aumentando a taxa de produção (Das e Butler, 1998; Boustani e Maini, 2001).

$\mathrm{O}$ objetivo do presente estudo consistiu em determinar o volume molar excesso, $V_{\mathrm{m}}^{\mathrm{E}}$, a partir de dados experimentais de densidade, de soluções liquidas binárias de glicerol + metanol, ou + etanol, ou + 1-propanol, ou + 2-propanol, ou + 1-butanol nas temperaturas de 288,15 K, 293,15 K, 298,15 K, 303,15 K e 308,15 K, em toda faixa de composição e à pressão atmosférica. Os resultados experimentais do volume molar excesso dos sistemas estudados foram correlacionados através de um polinômio de Redlich-Kister.

\section{PARTE EXPERIMENTAL}

Todos os reagentes utilizados no estudo apresentaram os seguintes valores de pureza, conforme especificações encontradas nos rótulos dos fabricantes: glicerol (Sigma-Aldrich, pureza $\geq 99,5 \%$ ), metanol (Merck, pureza $\geq 99,9 \%$ ), etanol (Merck, pureza $\geq 99,5 \%$ ), 1-propanol (Merck, pureza $\geq 99,8 \%$ ), 2-propanol (Merck, pureza $\geq 99,9 \%$ ) e 1-butanol (Merck, pureza $\geq$ 99,5\%). Nenhum dos reagentes passou por processo de purificação adicional. A Tabela 1 apresenta uma comparação entre os valores das densidades encontrados na literatura e os valores experimentais. 
Tabela 1 - Comparação entre as densidades experimentais, $\rho$, e aquelas encontradas na literatura dos componentes puros utilizados neste trabalho.

\begin{tabular}{lll}
\hline Componente & Literatura, $\rho^{293,15} / \mathrm{g} \cdot \mathrm{cm}^{-3}$ & Experimental, $\rho^{29,15} / \mathrm{g} \cdot \mathrm{cm}^{-3}$ \\
\hline Glicerol & 1,26088 (Egorov e Makarov, 2012) & 1,26080 \\
Metanol & 0,7915 (Pereira et al., 2003) & 0,79135 \\
Etanol & 0,789454 (Kijevcanin et al., 2007) & 0,78944 \\
1-Propanol & 0,80350 (Zarei et al., 2008) & 0,80343 \\
2-Propanol & 0,78513 (Zarei et al., 2008) & 0,78506 \\
1-Butanol & 0,80956 (Liau et al., 1998) & 0,81150 \\
\hline
\end{tabular}

As densidades dos componentes puros e das soluções binárias foram obtidas utilizando um densímetro de oscilação mecânica fabricado pela Anton Paar (modelo DMA 4500). A temperatura do tudo oscilador foi controlada automaticamente e mantida constante em $0,01 \mathrm{~K}$ através de um dispositivo eletrônico Peltier. $\mathrm{O}$ equipamento foi calibrado com ar e água. A incerteza na medida da densidade foi estimada em $2 \times 10^{-5} \mathrm{~g} \cdot \mathrm{cm}^{-3}$ e a incerteza no $V_{\mathrm{m}}^{\mathrm{E}}$ foi estimada em $1 \times 10^{-3} \mathrm{~cm}^{3} \cdot \mathrm{mol}^{-1}$. As amostras foram preparadas por pesagem dos reagentes, e para isso foi utilizada uma balança analítica fabricada pela OHAUS Adventurer (Modelo AR 2140, com resolução de $1 \times 10^{5} \mathrm{~g}$ ). A incerteza na fração molar foi estimada em $1 \times 10^{-4}$.

\section{RESULTADOS}

O volume molar excesso foi calculado a partir das densidades dos componentes puros e das respectivas soluções, através da seguinte equação:

$$
V_{\mathrm{m}}^{\mathrm{E}}=x_{1} M_{1}\left(\frac{1}{\rho}-\frac{1}{\rho_{1}}\right)+x_{2} M_{2}\left(\frac{1}{\rho}-\frac{1}{\rho_{2}}\right),
$$

onde $x_{i}$ representa a fração molar, $M_{i}$ é a massa molar, $\rho_{i}$ a densidade dos componentes puros e $\rho$ é a densidade da solução.

Os valores experimentais de $V_{\mathrm{m}}^{\mathrm{E}}$ foram ajustados através do seguinte polinômio de RedlichKister (Redlich e Kister, 1948):

$$
V_{\mathrm{m}}^{\mathrm{E}}=x_{2}\left(1-x_{2}\right) \sum_{j=\mathrm{o}}^{3} A_{j}\left(1-2 x_{2}\right)^{j} .
$$

Os valores do desvio padrão, $\sigma$, foram calculados utilizando a seguinte equação:

$$
\sigma=\left[\sum\left(V_{\exp }^{\mathrm{E}}-V_{\text {cal }}^{\mathrm{E}}\right)^{2} /(N-n)\right]^{1 / 2},
$$


onde $N$ é o número de dados experimentais e $n$ é o número de coeficientes do polinômio.

Os coeficientes $A_{j}$, obtidos pelo método dos mínimos quadrados, estão apresentados na Tabela 2, juntamente com os desvios padrões.

Tabela 2 - Coeficiente $A_{j}$ da Equação 2 e os desvios padrões, $\sigma$, calculados pela Equação 3, para os sistemas estudados a diferentes temperaturas.

\begin{tabular}{|c|c|c|c|c|c|}
\hline$T / \mathrm{K}$ & $A_{\mathrm{o}} / \mathrm{cm}^{3} \cdot \mathrm{mol}^{-1}$ & $A_{1} / \mathrm{cm}^{3} \cdot \mathrm{mol}^{-1}$ & $A_{2} / \mathrm{cm}^{3} \cdot \mathrm{mol}^{-1}$ & $A_{3} / \mathrm{cm}^{3} \cdot \mathrm{mol}^{-1}$ & $\sigma \times 10^{-2} / \mathrm{cm}^{3} \cdot \mathrm{mol}^{-1}$ \\
\hline \multicolumn{6}{|c|}{$x_{1} \mathrm{C}_{3} \mathrm{H}_{8} \mathrm{O}_{3}+\left(1-x_{1}\right) \mathrm{CH}_{3} \mathrm{OH}$} \\
\hline 288,15 & $-4,05526$ & $-1,89591$ & $-0,94453$ & $-1,30021$ & 1,132 \\
\hline 293,15 & $-4,21420$ & $-1,94683$ & $-1,06807$ & $-1,19480$ & 1,061 \\
\hline 298,15 & $-4,34670$ & $-2,01106$ & $-1,08433$ & $-1,23663$ & 1,034 \\
\hline 303,15 & $-4,48248$ & $-2,06893$ & $-1,10062$ & $-1,30284$ & 1,031 \\
\hline 308,15 & $-4,61902$ & $-2,12134$ & $-1,13228$ & $-1,38896$ & 1,018 \\
\hline \multicolumn{6}{|c|}{$x_{1} \mathrm{C}_{3} \mathrm{H}_{8} \mathrm{O}_{3}+\left(1-x_{1}\right) \mathrm{CH}_{3} \mathrm{CH}_{2} \mathrm{OH}$} \\
\hline 288,15 & $-3,73149$ & $-0,76020$ & $-0,54400$ & $-0,67345$ & 0,575 \\
\hline 293,15 & $-3,88854$ & $-0,79782$ & $-0,57280$ & $-0,44400$ & 0,278 \\
\hline 298,15 & $-4,00277$ & $-0,75733$ & $-0,51864$ & $-0,70646$ & 0,844 \\
\hline 303,15 & $-4,12065$ & $-0,78113$ & $-0,44776$ & $-0,78788$ & 0,891 \\
\hline 308,15 & $-4,23440$ & $-0,77279$ & $-0,38481$ & $-0,92178$ & 1,046 \\
\hline \multicolumn{6}{|c|}{$x_{1} \mathrm{C}_{3} \mathrm{H}_{8} \mathrm{O}_{3}+\left(1-x_{1}\right) \mathrm{CH}_{3}\left(\mathrm{CH}_{2}\right)_{2} \mathrm{OH}$} \\
\hline 288,15 & $-2,47349$ & 0,21987 & $-0,43685$ & $-0,41297$ & 1,100 \\
\hline 293,15 & $-2,59148$ & 0,22798 & $-0,51029$ & $-0,21574$ & 0,956 \\
\hline 298,15 & $-2,68279$ & 0,24021 & $-0,46250$ & $-0,22326$ & 0,903 \\
\hline 303,15 & $-2,77720$ & 0,26192 & $-0,41749$ & $-0,23002$ & 0,851 \\
\hline 308,15 & $-2,87880$ & 0,29327 & $-0,38257$ & $-0,24190$ & 0,904 \\
\hline \multicolumn{6}{|c|}{$x_{1} \mathrm{C}_{3} \mathrm{H}_{8} \mathrm{O}_{3}+\left(1-x_{1}\right) \mathrm{CH}_{3}\left(\mathrm{CH}_{2}\right)_{3}(\mathrm{OH})$} \\
\hline 288,15 & $-1,47130$ & 0,30552 & $-0,56124$ & 0,41225 & 0,653 \\
\hline 293,15 & $-1,56927$ & 0,33360 & $-0,63258$ & 0,55639 & 0,679 \\
\hline 298,15 & $-1,65004$ & 0,35427 & $-0,56764$ & 0,51938 & 0,638 \\
\hline 303,15 & $-1,73157$ & 0,40349 & $-0,56823$ & 0,48970 & 0,642 \\
\hline 308,15 & $-1,81779$ & 0,44377 & $-0,53409$ & 0,46943 & 0,644 \\
\hline \multicolumn{6}{|c|}{$x_{1} \mathrm{C}_{3} \mathrm{H}_{8} \mathrm{O}_{3}+\left(1-x_{1}\right) \mathrm{CH}_{3} \mathrm{CH}(\mathrm{OH}) \mathrm{CH}_{3}$} \\
\hline 288,15 & $-3,30930$ & 0,33579 & $-0,31593$ & 0,04677 & 1,176 \\
\hline 293,15 & $-3,46644$ & 0,34338 & $-0,36959$ & 0,22715 & 1,088 \\
\hline 298,15 & $-3,59903$ & 0,35236 & $-0,31764$ & 0,20897 & 1,085 \\
\hline 303,15 & $-3,74495$ & 0,35766 & $-0,26192$ & 0,20417 & 1,095 \\
\hline 308,15 & $-3,90641$ & 0,39132 & $-0,18128$ & 0,19511 & 1,146 \\
\hline
\end{tabular}




\section{DISCUSSÕES}

As Figuras 1-2 apresentam os valores do volume molar excesso, em função da fração molar do glicerol, a diferentes temperaturas. Para todos os sistemas estudados, $V_{\mathrm{m}}^{\mathrm{E}}$ é negativo em toda faixa de composição e temperaturas estudadas, e a contração segue a sequência: metanol > etanol $>$ 2-propanol > 1-propanol > 1-butanol. Isso também pode ser observado na Figura 3.

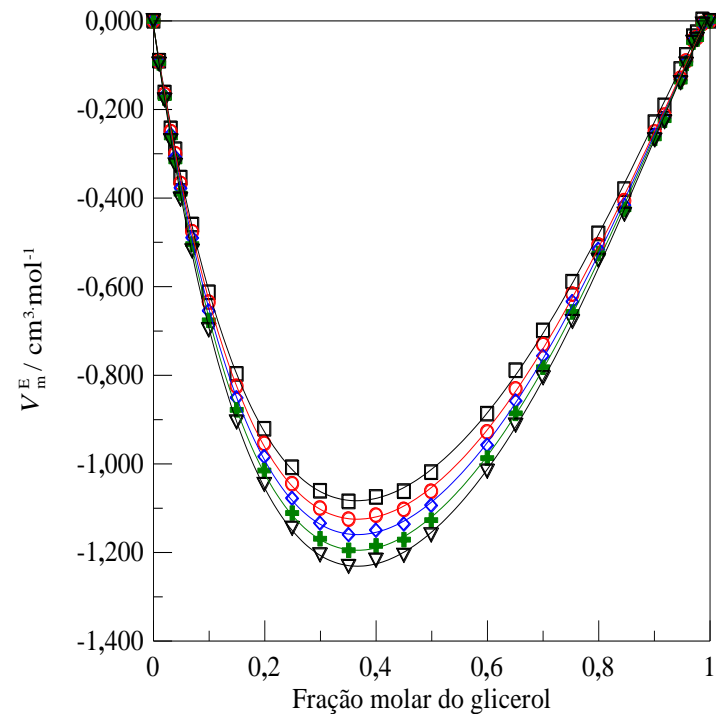

A

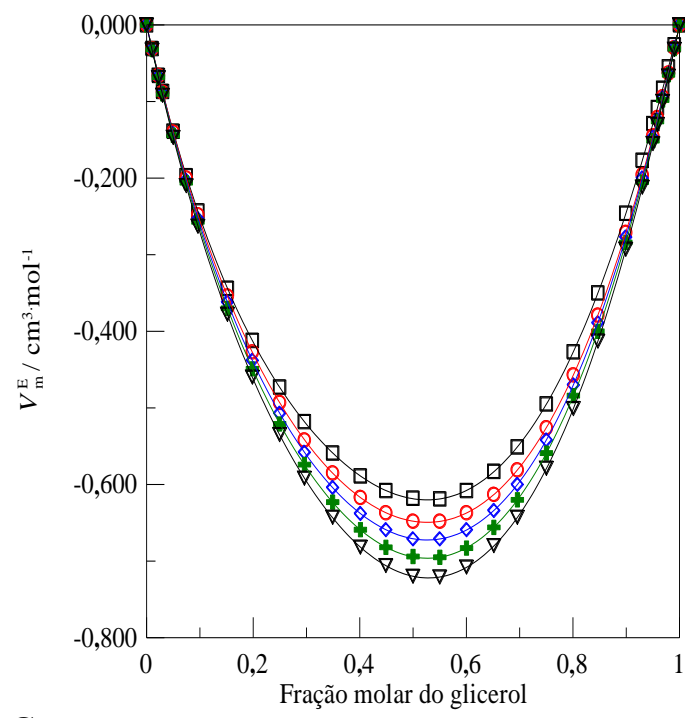

$\mathrm{C}$

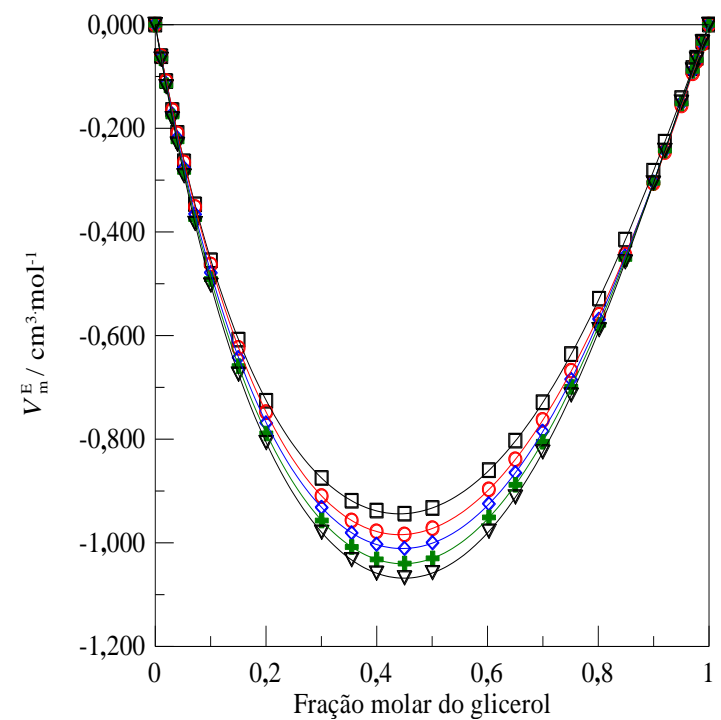

B

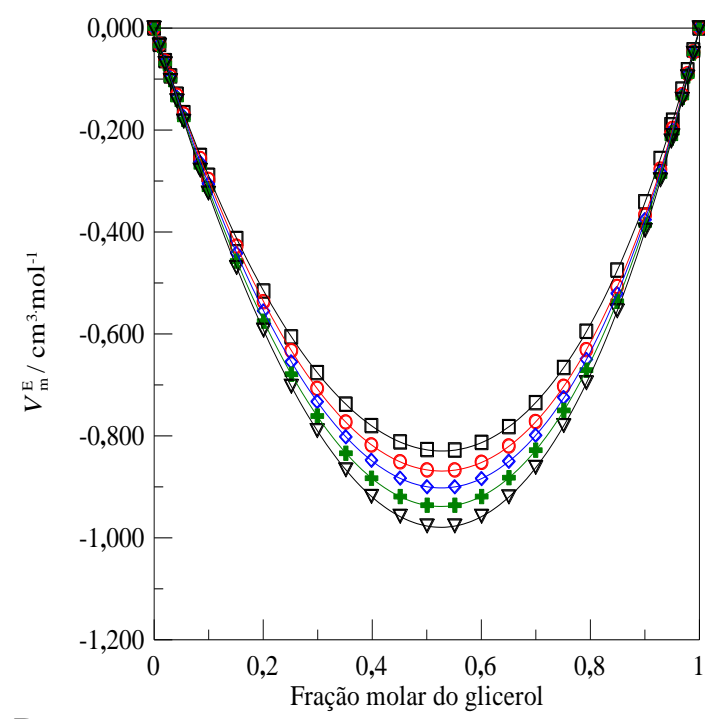

$\mathrm{D}$

Figura 1 - Volume molar excesso, em função da fração molar do glicerol, para o sistema $\left\{x_{1}\right.$ glicerol + (1 $-x_{1}$ ) álcool\} a diferentes temperaturas e à pressão atmosférica: $\square, 288,15 \mathrm{~K}$; $\bigcirc, 293,15 \mathrm{~K} ; \diamond, 298,15 \mathrm{~K}$; +, 303,15 K; $\nabla, 308,15 \mathrm{~K}:$ A, metanol; B, etanol; C, 1-propanol; D, 2-propanol. As linhas sólidas representam a correlação Redlich-Kister 


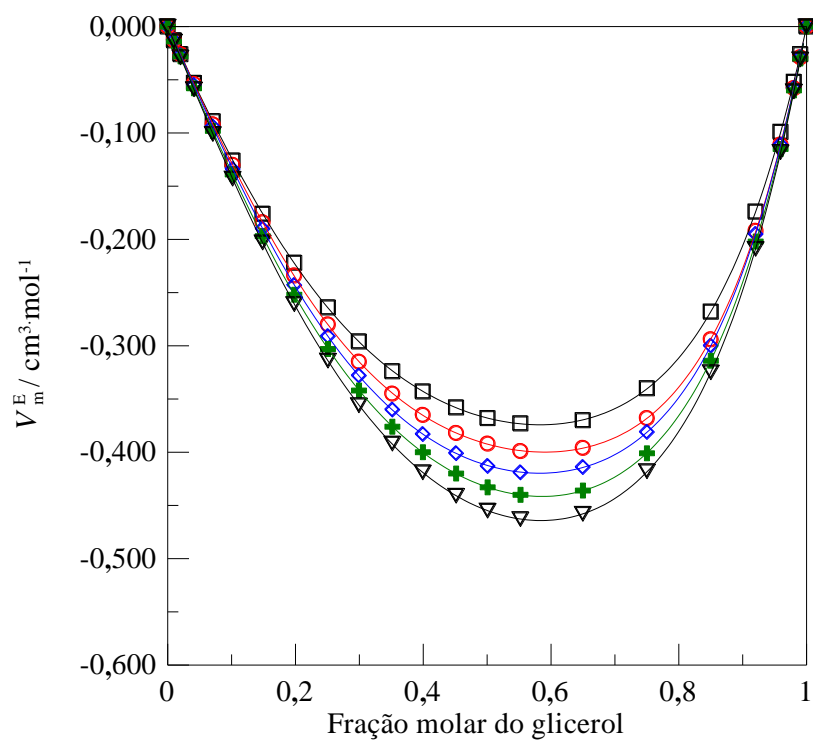

Figura 2 - Volume molar excesso, em função da fração molar do glicerol, para o sistema $\left\{x_{1}\right.$ glicerol + $\left(1-x_{1}\right)$ 1-butanol $\}$ a diferentes temperaturas e à pressão atmosférica: $\square, 288,15 \mathrm{~K} ; \bigcirc, 293,15 \mathrm{~K} ; \diamond$, $298,15 \mathrm{~K} ;++, 303,15 \mathrm{~K} ; \nabla, 308,15 \mathrm{~K}$. As linhas sólidas representam a correlação Redlich-Kister

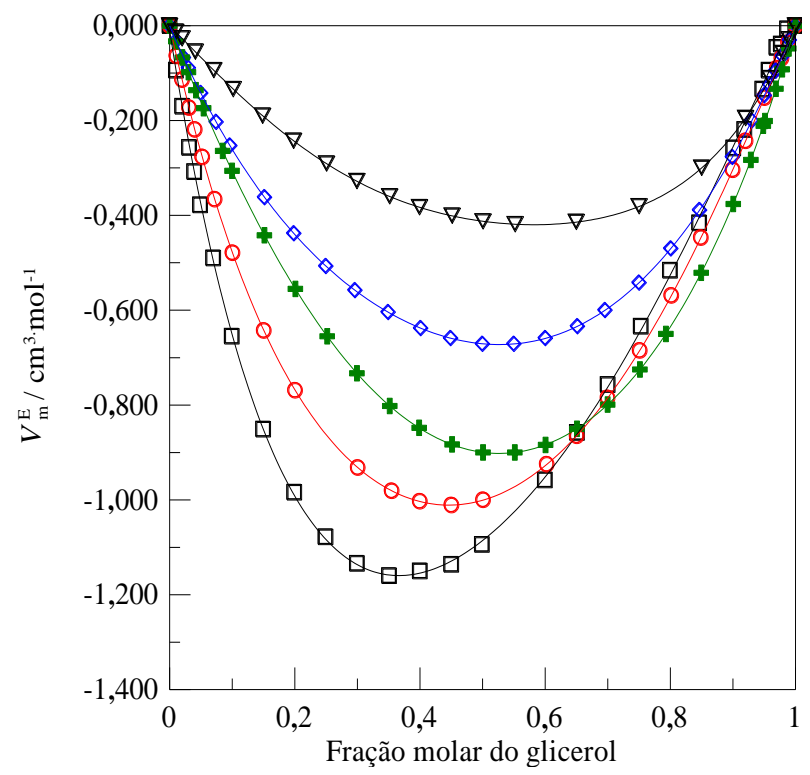

Figura 3 - Volume molar excesso, em função da fração molar do glicerol, a $T=298,15 \mathrm{~K}$ e à pressão atmosférica: $\square$, metanol; $\bigcirc$, etanol ; $\diamond, 1$-propanol; +, 2-propanol; $\nabla$, 1-butanol.

Valores negativos do volume molar excesso são atribuídos a dois principais fenômenos (Benson et al., 1981): (1) a efeitos estruturais decorrentes das acomadações das moléculas dos componentes presentes no sistema como consequeência da difernça entre os seus volumes 
molares e volumes livres; (2) a interações específicas, principalmente pela foramção de complexo de transferência de carga e ligações de hidrogênio entre as diferentes espécies químicas presentes no sistema. Para todos os sistemas estudados, o volume molar excesso aumentou em módulo, isto é, diminui com o aumento da temperatura. Uma vez que os efeitos químicos tendem a diminuir com o aumento da temperatura, é possível que os efeitos estruturais também sejam preponderantes no comportamento do $V_{\mathrm{m}}^{\mathrm{E}}$ para esses sistemas. A maior contração para o sistema contendo metanol pode ser entendida pela maior interação entre o glicerol e o metanol quando comparada aos outros alcoóis e pela melhor acomodação das moléculas do metanol na estrutura do glicerol. À medida que a cadeia carbônica cresce, esses efeitos tendem a diminuir.

\section{CONCLUSÕES}

No presente estudo, foram determinados dados experimentais de densidade de soluções líquidas binárias de (glicerol + alcoóis), em toda faixa de composição, a diferentes temperaturas e à pressão atmosférica. Os resultados experimentais foram usados para calcular o volume molar excesso dos sistemas. Os valores do $V_{\mathrm{m}}^{\mathrm{E}}$ foram negativos e seguiram a ordem: metanol > etanol > 2-propanol > 1-propanol > 1-butanol. O efeito da temperatura no comportamento do $V_{\mathrm{m}}^{\mathrm{E}} \operatorname{mostrou}$ que a contração aumenta com o aumento da temperatura. Os resultados do volume molar excesso foram correlacionados com um polinômio de Redlich-Kister. Os resultados experimentais obtidos neste estudo sugerem que as interações específicas e os efeitos estruturais devem prevalecer sobre os efeitos físicos.

\section{AGRADECIMENTOS}

Os autores agradecem a FEI e a Fundação de Amparo à Pesquisa do Estado de São Paulo (FAPESP) pelo auxilio financeiro através do Projeto 04/11855-8.

\section{REFERÊNCIAS}

BENSON, G.C.; HALPIN, C.J.; TRESZCZANOWICZ, A.J. Excess volumes and isentropic compressibilities for (2-ethoxyethanol + n-heptane) at 298.15 K. J. Chem. Thermodynamics, v. 13, p. 1175-1183, 1981.

BOUSTANI, A.; MAINI, B. The role of diffusion and convective dispersion in vapour extraction process. J. Can. Pet. Technol., v. 40, p. 68-77, 2001.

CALLAM, C.S.; SINGER, S.J.; LOWARY, T.L.; HADAD, C.M. Computational analysis of the potential energy surfaces of glycerol in the gas and aqueous phases: effects of level of theory, basis set, and solvation on strongly intramolecularly hydrogen-bonded systems. J. Am. Chem. Soc., v. 123, p. 11743-11754, 2001.

CHAMPENEY, D.C.; JOARDER, R.N.; DORE, J.C. Structural studies of liquid D-glycerol by neutrondiffraction. Mol. Phys., v. 58, p. 337-347, 1986. 


\section{9 a 22 de outubro de 2014 \\ Florianópolis/SC}

CHELli, R.; PROCACCI, P.; CARDINI, G.; DELLA VALLE, R.G.; CALIFANO, S. Glycerol condensed phases Part I. A molecular dynamics study. Phys. Chem. Chem. Phys., v. 1, p. 871877, 1999a.

CHELLI, R.; PROCACCI, P.; CARDINI, G.; CALIFANO, S. Glycerol condensed phases. Part II: a molecular dynamics study of the conformational structure and hydrogen bonding. Phys. Chem. Chem. Phys., v. 1, p. 879-885, 1999 b.

CHELlI, R.; GERVASIO, F.L.; GELLINI, C.; PROCACCI, P.; CARDINI, G.; SCHETTINO, V. Density functional calculation of structural and vibrational properties of glycerol. J. Phys. Chem., v. A104, p. 5351-5357, 2000.

DAS, S. K.; BUTLER, R. M. Mechanisms of the vapour extraction process for heavy oil and bitumen. J. Pet. Sci. Eng., v. 21, p. 43-59, 1998.

DASHNAU, J.L.; NUCCI, N.V.; SHARP, K.A.; VANDERKOOI, J.M. Hydrogen bonding and the cryoprotective properties glycerol/water mixtures. J. Phys. Chem., v. B110, p. 13670-13677, 2006.

DAWIDOWSKI, J.; BERMEJO, F.J.; FAYOS, R.; PEREA, R.F.; BENNINGTON, S.M.; CRIADO, A. Coherent neutron scattering response from glassy glycerol. Phys. Rev., v. E53, p. 5079-5088, 1996.

EGOROV, G.I.; MAKAROV, D.M. Volumetric properties of binary mixtures of glycerol + tertbutanol over the temperature range 293.15 to $348.15 \mathrm{k}$ at atmospheric pressure. J Solution Chem., v. 41, p. 536-554, 2012.

GARAWIA, M.; DOREA, J.C.; CHAMPENEY, D.C. Structural studies of liquid D-glycerol II. Molecular conformation and long range correlations. Mol. Phys., p. 62, v. 475-487, 1987.

KIJEVČANIN, M.L.; ŠERBANOVIĆ, S.P.; RADOVIĆ, I.R.; DJORDJEVIĆ, B.D.; TASIĆ, A.Ž. Volumetric properties of the ternary system ethanol + chloroform + benzene at temperature range (288.15-313.15) K: Experimental data, correlation and prediction by cubic EOS. Fluid Phase Equilib., v. 251, p. 78-92, 2007.

LIAU, W.-R.; TANG, M.; CHEN. Y.-P. Densities and viscosities of butyl acrylate + 1-butanol and ethyl laurate + 1-butanol at 293.15, 303.15, and 313.15 K. J. Chem. Eng. Data, v. 43, p. 826829, 1998.

MARCUS, Y. Some thermodynamic and structural aspects of mixtures of glycerol with water. Phys. Chem.Chem. Phys., v. 2, p. 4891-4896, 2000.

PEREIRA, S.M.; RIVAS, M.A.; LEGIDO, J.L.; IGLESIAS, T.P. Speeds of sound, densities, isentropic compressibilities of the system (methanol + polyethylene glycol dimethyl ether 250) at temperatures from 293.15 to 333.15K. J. Chem. Thermodynamics, v. 35, p. 383-391, 2003.

REDLICH, O.; KISTER, T. Algebraic representation of thermodynamic properties and the classification of solutions. Ind. Eng. Chemistry, v. 40, p. 345-348, 1948.

TOWEY, J.J.; SOPER, A.K.; DOUGAN, L. The structure of glycerol in the liquid state: a neutron diffraction study. Phys. Chem. Chem. Phys., v.13, p. 9397-9406, 2011.

ZAREI, H.A.; ASADI, S.; ILOUKHANI, H. Temperature dependence of the volumetric properties of binary mixtures of (1-propanol, 2-propanol and 1,2-propanediol) at ambient pressure (81.5 kPa). J. Molecular Liq., v. 141, p. 25-30, 2008.

VAN KONINGSVELD, H. The crystal structure of glycerol and its conformation. Recl. Trav. Chim. Pays-Bas., v. 87, p. 243-254, 1968. 